\title{
Improving Utilization of the Family History in the Electronic Health Record
}

Kathleen T. Hickey, EdD, RN, FAAN

[Corresponding Author]

Columbia University School of Nursing

617 W. $168^{\text {th }}$ St., Room 356

New York, NY 10032

Phone: 212-305-4944

Email: kth6@cumc.columbia.edu

Maria C. Katapodi, PhD, RN, FAAN

University of Basel Institute of Nursing Science

Bernoulli Street 28, Room 113

Basel, Switzerland 4056

Phone: +41 (0) 612670430

Email: maria.katapodi@unibas.ch

\section{Bernice Coleman, PhD, RN, FAAN}

Nursing Research and Development

North Tower, Room 2022

Cedars-Sinai Medical Center

8700 Beverly BLVD

Los Angeles, CA 90048

Phone: 310 423-6178

Email: Bernice.Coleman@cshs.org

Karin Reuter-Rice, PhD, RN, FAAN

Duke University School of Nursing

2059 Pearson Building

Durham, NC 27708

Phone: 919-681-7647

Email: karin.reuter-rice@duke.edu

\section{Angela R. Starkweather, PhD, RN, FAAN}

University of Connecticut School of Nursing

Augustus Storrs Hall

231 Glenbrook Road

Storrs, CT 06269

Phone: 860-486-0592

Email: angela.starkweather@uconn.edu 


\begin{abstract}
The importance of family health history is evident, and national efforts have been undertaken to improve documentation and the use of family history to improve health outcomes. Family health history is one of the most important tools for identifying the risk of developing rare and chronic conditions, including cardiovascular disease, cancer, and diabetes, and represents an integration of disease risk from genetic, environmental, and behavioral/lifestyle factors. In fact, family history has long been recognized as a strong independent risk factor for disease and is the current best practice used in clinical practice to guide risk assessment.

In this paper, we have identified opportunities to advance nursing contributions in obtaining, updating, and assessing family history in order to improve the health of all individuals. Identified opportunities are focused within the area of promoting the importance of communication within families and between healthcare providers to obtain, document, and update family histories. Nurses can increase awareness of existing resources that can guide collection of a comprehensive and accurate family history and facilitate family discussions. Aligned with the clinical preparation of nurses, family health should be used routinely by nurses for risk assessment and to help inform patient and family members on screening, health promotion, and disease prevention. The quality of family health information is critical in order to leverage the use of genomic healthcare information and derive new knowledge about disease biology, treatment efficacy, and drug safety. These actionable steps need to be performed in the context of promoting evidence-based applications of family history that will be essential for implementing personalized genomic healthcare approaches and disease prevention efforts.
\end{abstract}




\section{Introduction}

An accurate family history $(\mathrm{FH})$ is fundamental to providing the highest quality of personalized healthcare. Amidst advancements in genomic science and translation to improved diagnostic precision, the FH still provides the most efficient and low-cost potential of providing an individualized blueprint for care. In 2002, the Office of Public Health Genomics (OPHG) started the Family History Public Health Initiative to increase awareness of family health history as an important risk factor for disease and to promote the use of $\mathrm{FH}$ in programs aimed at reducing the burden of disease in the U.S. population (Centers for Disease Control and Prevention, 2013). This was followed in 2009, by the National Institutes of Health State of the Science Statement, which aimed to provide health care providers, patients, and the general public with an assessment of currently available data on FH and improving health (NIH, 2009). Although a comprehensive, 3-generation pedigree used in medical genetics, counseling, and research remains a recognized important assessment for disease prevention and health promotion, significant knowledge gaps exist (Guttmacher, Collins, \& Carmona, 2004; Valdez, Yoon, Qureshi, Green \& Khoury, 2010; Pyeritz, 2012). Since the original publication of the National Institutes of Health State of the Science Statement, the following gaps continue to exist:

(1) Lack of standardized practices for documenting and updating the FH given existing time and resource restraints in our healthcare system;

(2) Lack of ability to access family health data across the many different health care settings by all members of the inter-professionals team where services are obtained, and ideally, coordinated (e.g., hospitals, home, outpatient settings, clinics, schools, communities);

(3) Inability to incorporate non-biological family members who share common social-behavioral, cultural and environmental factors;

(4) Inability to link family histories, which are dynamic and ever changing, with genomic data to guide automated risk assessment, prevention and health promotion strategies within electronic health information systems; 
(5) Lack of easy to use, culturally, linguistically, and educationally tailored resources, which can guide families on how to start the conversation on family health and support in acquiring, documenting, and updating a family history;

(6) Lack of a cohesive education strategy to support inter-professional communications of the health team's ability to translate genomic finding into practice.

The authors and members of The American Academy of Nursing's Genetic Nursing and Healthcare Expert Panel sought to further explore the current state-of-evidence on these issues in order to identify a process for advancing the utilization of FH in clinical practice. Nurses have an important role in promoting family health tracking among patients and families, the acquisition and documentation of a three-generation $\mathrm{FH}$ in the electronic health record (EHR) and performing updates of family health information and risk assessment on a routine basis for every individual.

For this to happen consistently across healthcare systems, action must be taken to: (1) standardize collection methods and documentation standards; (2) ensure return of evidence-based recommendations to promote adoption of health promotion behaviors; and (3) advance interoperability of FH data among individuals, clinicians, and healthcare systems.

$\underline{\text { Standardized Family Health Collection Methods and Documentation Standards }}$

Nurses have an important role in promoting family discussion and documentation of $\mathrm{FH}$. Numerous agencies have provided recommendations on how to begin discussions about FH, including a tool developed by the U.S. Surgeon General, My Family Health Portrait (https://familyhistory.hhs.gov/FHH/html/index.html). Advantages of using these tools include wider dissemination of health information among family members and clinicians and less time required to collect the information in the healthcare setting. However, these strategies have not been routinely adopted into the delivery of healthcare, largely due to the potential inaccuracies of the information (Facio, Feero, Linn, Oden, Manickam, \& Biesecker, 2010). To address this issue, metrics for the quality of FH data were developed and tested using MeTree, a patient-facing webbased family and personal health history collection and clinical decision support program (Wu et 
al., 2014). While using MeTree improved the quantity and quality of data collected, the study also found that the process of engaging patients to discuss $\mathrm{FH}$ with relatives prior to collection improved the quantity and quality of data provided by patients.

Recommendations for using specific FH tools and core FH data sets have been published (Feero, Bigley, Brinner, \& The Family Health History Multi-Stakeholder Workgroup of the American Health Information Community, 2008). However, the lack of a standardized process for charting FH remains a significant obstacle to implementation. A strategy to promote quality FH data within the EHR interface would be implementation of metrics that must be routinely updated in order to qualify as complete documentation, such as those defined by Wu and colleagues (2014), including: (1) three generations of relatives; (2) relatives' lineage; (3) relatives' gender; (4) an up-to-date FH; (5) pertinent negatives noted; (6) age of disease onset in affected relatives; and for deceased relatives, the (7) age and (8) cause of death. This process would necessitate a reconceptualization of how FH is charted; instead of having one free-text box to fill in, the FH would be documented by filling in all known metrics.

Thus, the process of including comprehensive FH in EHR's can facilitate family discussions about family health; improve communication among individuals and healthcare provider, as well as the verification of FH data. One method of promoting wider adoption of FH tools in the EHR is application program interface (API), which allows data transfer between EHR platforms. A verification protocol, similar to the process used by Wikipedia that tags verified/unverified information, could be used in the EHR to improve confidence in, and utilization of, FH. In addition, automated reminders in the EHR should be included to remind the healthcare team to ask for updates in the $\mathrm{FH}$, flag data for further investigation or screening, and to link health information from other family members in the system. Automated updates of FH information may enhance the process of data collection if family member profiles are linked (e.g., a confirmed diagnosis in a father or mother would be automatically updated in their child's profile). In children, automatic FH updates could also cross-pollinate into siblings histories thereby drawing attention to familial risk or exposure. 
Ensure Return of Evidence-based Recommendations to Promote Adoption of Health Promotion Behaviors

Obtaining an accurate three-generation FH is one critical area in which nurses and other healthcare professionals can actively engage. However, FH continues to be under-documented and under-utilized in clinical practice. Retrospective reviews of EHRs have continued to show that $\mathrm{FH}$ information is gathered for only a small percentage of patients and lacks sufficient detail for an accurate risk assessment (Langlands, Prentice \& Ravine, 2010; Qureshi et al., 2009). Beyond the barrier of lack of time to accurately collect, update and document FH data, there is also a lack of access to guidelines or resources for estimating the impact of FH (Daelemans, Vandevoorde, Vansintejan, Borgermans, Devroey, 2013). Documentation of FH in at least 20\% of patients is an optional "menu-set objective" that healthcare providers may use to meet Stage 2 meaningful use requirements for the Centers for Medicare and Medicaid Service's (CMS) electronic health record incentive program. However, this requirement falls short of its mandate by the lack of criteria for quality assessment (e.g., documenting all chronic conditions and reason/age of morbidity in three generations) and evaluation of risk, that is, the utilization of the data to provide recommendations for health promotion and disease prevention. Ideally, automated messages could be designed in the EHR to flag patients at risk of health conditions based on FH data; however, the implementation of this approach requires provider engagement. Recently, a study that evaluated primary physician responses to automated tailored prompts that alerted them to a patient's risk to one or more of six diseases based on FH found no significant change in adding the family history risk to the problem summary lists or screening interventions (Zozove, Plegue, Uhlmann, \& Ruffin, 2015). In addition, the manner in which healthcare providers are reimbursed for services, such as the time and effort for collecting and documenting a family history, has yet to be addressed. Thus, better strategies of integrating the collection and utilization of FH data are needed.

Educational standards of bachelor's level nurses include the basic competency of obtaining a family health history and provision of basic risk assessment information to individuals and families. Master's and doctoral level nurses are prepared to manage risks identified in the family history in order to improve health outcomes. Through collaboration with 
the American Nurses Association (ANA) and the International Society of Nurses in Genetics (ISONG), genetic and genomic competencies and outcomes have been defined for nursing across all levels of education (Consensus Panel, 2006). While a national strategy to support the integration and translation of genetics and genomics has been defined for nursing (Calzone, 2013), the competencies would ideally extend across all inter-professional team members in order to facilitate communication and outcomes.

Limited evidence exists on the effect of family history collected by nurses on the delivery of healthcare services and the impact on direct and indirect health outcomes. Future integrated electronic healthcare delivery systems, particularly those utilizing EHRs, may provide greater opportunities to evaluate the role of nursing in collecting, documenting, updating, and applying the $\mathrm{FH}$ at all levels of practice across all populations

Nurses work in many settings and are the most trusted healthcare professionals; thus, they are ideally suited to answer many important questions related to the FH. This includes questions related to the variance in accuracy and completeness of FH information according to the setting, mode of collection, and the person who is collecting it. In addition, nurses are able to recognize and assess how genomic health literacy, family dynamics, and various health disorders affect an individual's awareness and ability to communicate and report their family health history.

Nurses are also uniquely positioned to identify and answer other important questions that will impact personalized healthcare. These questions may pertain to which environmental and lifestyle elements of a family history are most useful in helping patients make positive changes in health-related behavior. Nurses may also inform how FH may be best collected and integrated into practice in diverse racial, ethnic, religious, social, cultural, and economic populations. Finally, simple to use "real world" culturally, linguistically, and educationally tailored resources that will allow nurses and other healthcare professionals to accurately update, assess and make evidence-based recommendations across healthcare settings are needed to make this a reality. Nurses are ideally suited to develop FH resources for implementation across a variety of healthcare settings.

The Family History Impact Trial Group has provided critically important data for informing the ways in which we help patients engage in efforts to promote and/or maintain 
health. Using a self-administered web-based tool, Family Healthware, that provides personalized risk-tailored messages, they examined the influence on physical activity, fruit and vegetable consumption, and cholesterol screening (Ruffin et al., 2011). Six-months after implementation of tailored messages, they found a modest increase in self-reported physical activity and fruit and vegetable intake, but reduced likelihood of receiving cholesterol screening. Although this study did not demonstrate an appreciable improvement in health outcomes or behavior change, other studies have been focused on how technology can motivate adoption of health promotion behaviors (Joseph, Keller, Adams, \& Ainsworth, 2015; Nahm et al., 2015).

Nurses are often the most readily available healthcare team member and routinely spend more time with patients and families regarding their health. The delivery of evidence-based recommendations must occur within the context of the patient and family relationship with consideration of environment and resources. Advances in understanding the importance of epigenetics in health has brought an increasing interest in incorporating FH elements to include fictive kin and other-mothers, which are non-biological family members that play a major role in the transmission of culture, health promotion, and decision-making (Spruill, Coleman, PowellYoung, Williams, \& Magwood, 2014). With this knowledge, there is greater potential to identify at-risk individuals and provide opportunities for education, prevention, and early diagnosis. As common diseases cluster within ethnic families as a result of shared environment and genetics, there has also been an interest in including neighbors and communities in the family health risk assessment (Hartmann, Marshall, \& Goldenberg, 2015). Housing and neighborhood factors may also be included as social and behavioral determinants of health (SBDH), indices that are also recommended for inclusion in the EHR by the Academy, the Agency for Healthcare Research and Quality (AHRQ), and the World Health Organization (WHO) (http://www.nursingoutlook.org/article/S0029-6554(15)00257-2/fulltext; http://www.healthypeople.gov/2020/topics-objectives/topic/social-determinants-health; http://www.who.int/social determinants/en/). As similar challenges are inherent in collecting, analyzing, and providing recommendation for $\mathrm{FH}$ and SBDH factors, it may be reasonable to address these issues together. Nurses are in a prime position to take a leading role in the 
implementation process for ensuring that this data is collected and addressed within the realms of healthcare for individuals and families.

\section{Integrating Family Health History with Other Genomic Tools}

The potential for having an EHR that is available to patients across their lifespan and accessible to healthcare providers during every interaction with the healthcare system is becoming more of a reality. As genetic and genomic testing is becoming more readily available and more frequently used by healthcare providers, there is great potential for this data to be housed within individual EHR profiles. This advancement could provide another avenue for linking FH data with genomic risk factors and improving the early diagnosis and treatment of common chronic health conditions.

Nurses across the nation are performing cutting-edge research to identify genomic risk factors and gene $\mathrm{x}$ environment interactions that influence health outcomes. With increasing proficiency in the utilization and interpretation of big data, nurses will continue to provide personalized health interventions and contribute to President Obama's Precision Medicine Initiative ${ }^{\circledR}(\mathrm{PMI})$ announced in January 2015 (https://www.whitehouse.gov/the-pressoffice/2015/01/30/fact-sheet-president-obama-s-precision-medicine-initiative). The integration of family health history with genetic test results will be a major issue that needs to be addressed as EHRs provide the capability of automating the analysis of genomic discoveries into personalized risk stratification. As technology continues to increase the ability to advance precision approaches for health, nurses must be prepared to interpret and discuss this information with individuals and families.

While some of these systems are becoming more automated, patients will still require assistance with knowing how to apply the information in the context of screening recommendations and options for addressing psychological, behavioral, and/or environmental risk factors. In a recent study using MeTree, the number of patients at increased risk for breast/ovarian cancer, colon cancer, hereditary syndrome risk and thrombosis were examined (Orlando et al., 2014). The authors reported that the implementation of risk stratification tools in primary care will likely increase costs related to an increased prevalence of non-routine risk 
assessment and management resources required. As tools, such as MeTree, become more available, nurses are ready to be part of the solution in assisting patients and families to make the right personal choices. Nurses play an important role as frontline educators and advocates of patients and families, and documentation of FH and risk assessment should be part of routine practice.

Family history remains the best means of assessing lifespan health risks and for providing patients and families with the best guidance on lifestyle choices to prevent chronic disease. Incorporating genomic risk assessments with other genomic advancements using a lifespan approach will only increase the ability of nurses to provide more precise information to patients and families and provide more momentum to improve the health of the nation.

\section{Conclusions}

In order to further its mission of identifying trends and issues important to healthcare delivery and policy, we have identified opportunities to advance the contributions of nurses in obtaining, updating and assessing family history in order to improve the health of all individuals and populations. Identified opportunities are focused within the areas of:

- urging Medicare and healthcare insurance companies to adopt quality metrics of FH data as an essential component of documentation; routine risk assessment should be addressed in health promotion and screening recommendations for each patient encounter

- promoting the importance of communication within families and between healthcare providers to obtain, document and update family histories at all levels of practice;

- improving the awareness and utility of existing resources and developing new resources that can guide and increase the frequency of obtaining and updating a comprehensive and accurate family history;

- developing and evaluating new tools that can be used in primary care and nontraditional settings that will integrate family history with other genomic healthcare 
information to derive new knowledge about disease biology, risk assessment, treatment efficacy, and drug safety;

- promoting evidence-based applications of family history for implementing personalized genomic health care approaches and disease prevention efforts;

- determining the impact of family history on direct and indirect health outcomes, patient choices, economic healthcare costs across the lifespan through systematic evaluation; and

- developing strategies that assist families to understand the value of passing generational information on within families and with health care providers as a mechanism to inform and maintain health through personal engagement.

\section{Acknowledgements: We would like to thank the members of The American Academy of Nursing's Genetic Nursing and Healthcare Expert Panel, Matthew Williams, and Ellen Olshansky for their input on this manuscript.}

\section{Clinical Resources:}

http://www.hhs.gov/programs/prevention-and-wellness/family-health-history/index.html https://www.healthit.gov/providers-professionals/achieve-meaningful-use/menu-measures2/family-health-history

http://www.isong.org/

http://geneticalliance.org/programs/genesinlife/fhh

https://phpartners.org/public_health_genomics.html

https://www.cdc.gov/genomics/famhistory/ 


\section{References}

American Congress of Obstetricians and Gynecologists (2011). Family history as a risk assessment tool. Accessed 10/21/2015 from http://www.acog.org/Resources-And$\underline{\text { Publications/Committee-Opinions/Committee-on-Genetics/Family-History-as-a-Risk- }}$ $\underline{\text { Assessment-Tool }}$

American Nurses Association. (2009). Genetics and Genomics Nursing: Competencies, curricula guidelines, and outcome indicators ( $2^{\text {nd }}$ ed.). Silver Springs, MD: American Nurses Association.

Carmona, R. H. (2004). The family history-more important than ever. New England Journal of Medicine, 351, 2333-2336.

Calzone, K. A., Jenkins, J., Bakos, A. D., Cashion, A. K., Donaldson, N., Feero, W. G...Webb, J. (2013). A blueprint for genomic nursing science. Journal of Nursing Scholarship, 45, 96104.

Centers for Disease Control and Prevention. (2013). Genomics translation. Retrieved on 11/5/2014 from http://www.cdc.gov/genomics/famhistory/famhist.htm

Daelemans, S., Vandevoorde, J., Vansintejan, J., Borgermans, L., \& Devroey, D. (2013). The use of family history in primary health care: a qualitative study. Advances in Preventative Medicine, Article ID 695763.

Facio, F. M., Feero, W. G., Linn, A., Oden, N., Manickam, K., \& Biesecker, L. G. (2010). Validation of My Family Health Portrait for six common heritable conditions. Genetics in Medicine, 12(6), 37-375. 
Feero, W. G., Bigley, M. B., Brinner, K. M., \& The Family Health History Multi-Stakeholder Workgroup of the American Health Information Community. (2008). New standards and enhanced utility for family health history information in the electronic health record: an update from the American Health Information Community’s Family Health History Multi-Stakeholder Workgroup. JAMA, 15(6), 723-728.

Hartmann, C. D., Marshall, P. A., \& Goldenberg, A. J. (2015). Is there a space for place in family history assessment? Underserved community views on the impact of neighborhood factors on health and prevention. Journal of Primary Prevention, 36, 119130.

Joseph, R. P., Keller, C., Adams, M. A., \& Ainsworth, B. E. (2015). Print versus a culturallyrelevant Facebook and text message delivered intervention to promote physical activity in African American women: a randomized pilot trial. BMC Womens Health, 15, 30.

Langlands, A. R., Prentice, D. A., \& Ravine, D. (2010). A retrospective audit of family history records in short-stay medical admissions. $M J A, 192(12), 682-684$.

Nahm, E. S., Resnick, B., Bellantoni, M., Zhu, S., Brown, C., Brennan, P. F...Plummer, L. (2015). Dissemination of a theory-based online bone health program: two intervention approaches. Health Informatics, 21(2), 120-136.

National Institutes of Health. (2009). NIH state-of-the-science conference statement on family health history and improving health. Bethesda, MD: National Institutes of Health.

O'Neill, S. M., Rubinstein, W. S., Wang, C., Yoon, P. W., et al. (2009). Familial risk for common diseases in primary care: the Family Healthware Impact Trial. American Journal of Preventative Medicine, 36(6), 506-14. 
Orlando, L. A., Wu, R. R., Beadles, C., Himmel, T., Buchanan, A. H., Powell, K. P...Ginsburg, G. S. (2014). Implementing family health history risk stratification in primary care: impact of guideline criteria on populations and resource demand. American Journal of Medical Genetics Part C, 166C, 24-33.

Pyeritz, R. E. (2012). The family history: the first genetic test, and still useful after all those years? Genetic Medicine, 14, 3-9.

Ruffin, M. T., Nease, Jr., D. E., Sen, A., Pace, W. D., Wang, C., Acheson, L. S...the Family History Impact Group. (2011). Effect of preventive message tailored to family history on health behaviors: The Family Healthware Impact Trial. Annals of Family Medicine, 9, 311.

Quereshi, N., Wilson B., Santaguida, P., Little, J., Carroll, J., Allanson, J., \& Raina, P. (2009). Family history and improving health. Evidence report/technology assessment, 186, 1135.

Spruill, I. J., Coleman, B. L., Powell-Young, Y. M., Williams, T. H., \& Magwood, G. (2014). Non-biological (fictive kin and othermothers): embracing the need for a culturally appropriate pedigree nomenclature in African-American families. Journal of the National Black Nurses Association, 25(2), 23-30.

Valdez, R., Yoon, P. W., Qureshi, N., Green, R. F., Khoury, M. J. (2010). Family history in public health practice: a genomic tool for disease prevention and health promotion. Annual Review of Public Health, 31, 69-87.

Wang, C., O'Neill, S. M., Rothrock, N., Gramling, R., et al. (2009). Comparison of risk perceptions and beliefs across common chronic diseases. Preventative Medicine, 48(2), $197-202$. 
Wu, R. R., Himmel, T. L., Buchanan, A. H., Powell, K. P., Hauser, E. R., Ginsburg, G. S., Henrich, V. C., \& Orlando, L. A. (2014). Quality of family history collection with use of a patient facing family history assessment tool. BMC Family Practice, 15, 31.

Yoon, P. W., Scheuner, M. T., Jorgensen, C., Khoury, M. J. (2009). Developing Family Healthware, a family health history screening tool to prevent common chronic diseases. Prevention of Chronic Disease, 6(1), A33.

Zazove, P., Plegue, M. A., Uhlmann, W. R. \& Ruffin, M. T. $4^{\text {th }}$. (2015). Prompting primary care providers about increased patient risk as a result of family history: Does it work? Journal of the American Board of Family Medicine, 28(3), 334-342. 